\title{
Tabaquismo entre universitarios: caracterización del uso en la visión de los estudiantes
}

\author{
Claudia María Sánchez-Hernández ${ }^{1}$ \\ Sandra Cristina Pillon ${ }^{2}$
}

\begin{abstract}
El objetivo fue conocer los significados atribuidos al consumo de tabaco entre estudiantes universitarios recién ingresados en la Universidad de Tegucigalpa, Honduras. Se trata de un estudio cualitativo con referencial metodológico fundamentado en el interaccionismo simbólico. Se formaron cuatro grupos focales (10 a 12) de estudiantes de distintas carreras, de ambos sexos, fumadores y no fumadores. Las principales categorías de significados acerca del consumo de tabaco fueron la presencia de fumadores en las familias de origen y los espacios para la iniciación del consumo de tabaco. Entre las razones para el inicio en el consumo de tabaco se encontraron factores como influencia de amigos y compañeros de trabajo. La práctica de deportes y los compromisos personales fueron atribuidos como factores no favorables al uso de tabaco. Se concluye que la universidad es un espacio abierto para el desarrollo de la promoción de estrategias educativas y preventivas frente al uso de drogas.
\end{abstract}

Descriptores: Estudiantes; Tabaquismo / Prevención y Control.

\footnotetext{
${ }^{1}$ Profesora, Carrera de Orientación Educativa, Universidad Pedagógica Nacional Francisco Morazán, Tegucigalpa, Honduras. E-mail: claudiamariasan@yahoo.es.

2 Enfermera, Doctora en Enfermería. Profesor Asociado, Escola de Enfermagem de Ribeirão Preto, Universidade de São Paulo, Centro Colaborador de la OMS para el Desarrollo de la Investigación en Enfermería, SP, Brasil. E-mail: pillon@eerp.usp.br.
}

Correspondencia:

Sandra Cristina Pillon

Universidade de São Paulo. Escola de Enfermagem de Ribeirão Preto

Departamento de Enfermagem Psiquiátrica e Ciências Humanas

Av. dos Bandeirantes, 3900

Bairro: Monte Alegre

CEP: 14040-902 Ribeirão Preto, SP, Brasil

E-mail: pillon@eerp.usp.br 


\section{Tabagismo entre universitários: caracterização do uso na visão dos estudantes}

O objetivo foi conhecer os significados atribuídos ao consumo de tabaco entre estudantes universitários de primeiro ano, da universidade de Tegucigalpa, Honduras. Trata-se de estudo qualitativo, e como referencial metodológico fundamentou-se no interacionismo simbólico. Realizaram-se quatro grupos focais (10 a 12) de estudantes de cursos distintos, de ambos os sexos, fumantes e não fumantes. As principais categorias de significados, a respeito do consumo de tabaco, foram presença de fumantes nos famílias de origem e os espaços para o início do consumo de tabaco. Entre as razões para o início do consumo de tabaco, foi indicada a influência de amigos e companheiros de trabalho. A prática de esportes e os compromissos pessoais foram atribuídos como fatores que não favorecem o consumo de tabaco. Concluí-se que a universidade é espaço aberto para o desenvolvimento e a implantação de estratégias educativas no âmbito da prevenção ao uso de tabaco.

Descritores: Estudantes; Tabagismo / Prevenção \& Controle.

\section{Smoking Among College Students: Characterization of Use in the Students' Perspective}

The aim was to determine the meanings attributed to tobacco consumption among freshmen college students at the University of Tegucigalpa, Honduras. A qualitative study was carried out, using symbolic interactionism as the methodological framework. We conducted four focus groups with 10 to 12 male and female students from different courses, smokers and nonsmokers. The main categories of meanings regarding tobacco use were the presence of smokers in families of origin, the spaces for the start of smoking. The reasons for the initiation of tobacco use include influence from friends and colleagues. Sports and personal commitments were considered factors that do not promote tobacco use. We concluded that the university is an open space for the development and implementation of educational strategies in the prevention against tobacco use.

Descriptors: Students; Smoking / Prevention \& Control.

\section{Introducción}

El consumo de tabaco es la epidemia que más causa mortalidad en el mundo. Por su aumento y extensión se considera una pandemia, cuyo incremento se relaciona actualmente con la publicidad. El consumo es tolerado y fomentado por la mayoría de los gobiernos, debido principalmente a los ingresos económicos que derivan de la producción y comercialización del tabaco, sin considerar que cerca de diez mil personas mueren al día por causa del humo del cigarrillo(1). Asimismo, se están mostrando altas repercusiones en el ámbito mundial, en la salud de la población de distintas edades. Diariamente, el número de personas que presentan consumo de tabaco está aumentando a pesar de los esfuerzos que las organizaciones públicas y privadas llevan a cabo para desarrollar labores de prevención y rehabilitación ${ }^{(2)}$.

El tabaco no es peligroso sólo para quien lo consume en forma de cigarrillo, lo es también para quienes inhalan el humo del tabaco medioambiental(1) denominado actualmente humo de segunda mano, el cual es reconocido como causante de numerosas patologías en personas no fumadoras expuestas al humo de su medio ambiente ${ }^{(2)}$.

La nicotina es una droga estimulante que incita al sistema nervioso simpático y produce sensaciones de optimismo, energía ilimitada, su empleo puede ser muy reforzador y el potencial de abuso se incrementa(3). 
El tabaquismo es definido como una enfermedad adictiva cuyo agente productor es la nicotina. En cuanto a la distinción entre fumadores y no fumadores, conceptualmente el no fumador es aquella persona que nunca ha consumido cigarrillos. En tanto el fumador es quien ha consumido cigarrillos por lo menos una vez en su vida(4).

Los obstáculos encontrados en la lucha contra el tabaquismo son múltiples y creativos, por ejemplo las campañas comerciales para apalear el consumo entre los jóvenes, las que presentan el consumo de drogas como algo atractivo y natural. Su influencia es continua y persistente contrarrestando las iniciativas que buscan aminorar el porcentaje de población que es consumidora regular del tabaco. El camino por recorrer aún es arduo, ya que el hábito nocivo de fumar, encuentra nuevos seguidores de todas las edades, con resultados que pueden ser letales. Por tanto, su constante afrontamiento requiere de estrategias que ultrapasen los límites de las fronteras nacionales. Esta realidad es comparable con lo que sucede en América Latina en países como Brasil, Colombia, Perú y en los países que integran el istmo centroamericano, cuya ubicación geográfica los coloca en una posición estratégica para ser corredor de distribución de drogas.

La Organización Panamericana de la Salud (OPS) ha advertido sobre las trágicas consecuencias que implica la adicción al tabaco; reporta que la mitad de las personas mueren de alguna enfermedad directamente relacionada con el consumo de nicotina y otros componentes nocivos.

En el caso de Honduras, sus habitantes poco a poco van tomando conciencia acerca del daño que ocasiona, a la salud personal y a la colectiva, el uso del tabaco. La edad de inicio es de 8 años, un tercio de los hondureños fuma regularmente. En 2005, Honduras fue incluido por la Organización Mundial de la Salud, junto a países como Canadá, México, Panamá, Perú y Trinidad y Tobago, como un país que desarrolla iniciativas para disminuir $y$ erradicar el fenómeno de las drogas(5).

La universidad como institución de educación superior se considera un espacio abierto, de libre ingreso a sus instalaciones, en la cual concurre una cantidad significativa de personas ubicadas principalmente en el estrato de edad adulta joven. Por tanto, no ha escapado, al igual que otros centros educativos de nivel superior al flagelo del fenómeno de drogas, siendo notorio observar la ingesta de tabaco entre sus miembros. A pesar de que se ejecutan programas encaminados a la promoción de estilos de vida saludables con el propósito de motivar a la comunidad universitaria a reducir su práctica de consumo de estas drogas lícitas y socialmente aceptadas, el impacto ha sido mínimo tomando en cuenta la cantidad de población que se reúne diariamente en la misma.

Por ello, es importante señalar el compromiso social de las universidades con el desarrollo humano sustentado en lo que debería ser, definido como una perspectiva de función social y de desarrollo, comprendido como un proceso de cambio progresivo de calidad de vida de los seres humanos-sujetos esenciales y objeto de desarrollo, por medio del crecimiento económico con equidad social, que preserve el patrimonio cultural y el equilibrio ecológico, de manera de asegurar la calidad de vida de las futuras generaciones ${ }^{(6)}$.

El primer año de universidad, particularmente las primeras semanas, es a menudo el tiempo de establecer la propia identidad y encontrar nuevos amigos. Entrar en el amplio ambiente de la universidad, lo cual ya no es limitada por los miembros de un grupo de pares envolventes que marcaron los años de educación secundaria. Es relevante notar que el proceso de transición hacia la vida universitaria es afrontada con una gran parte del tiempo no estructurado y pocas reglas explicitas que gobiernen su conducta ${ }^{(7)}$.

En la actualidad, se evidencia una notable preocupación sobre el consumo de tabaco entre los jóvenes universitarios. Un estudio realizado en una universidad peruana acerca de las prácticas de consumo de tabaco y otras drogas en 2,074 estudiantes de pregrado reveló que la edad de inicio del consumo de tabaco ocurre en la adolescencia, la prevalencia de vida y el uso actual de tabaco fueron de $81.9 \%$ y $38.7 \%$. Con una fuerte asociación entre el hábito de fumar de padres, hermanos y amigos y el hecho de ser o no fumador ${ }^{(4)}$. De manera que al iniciar la universidad los jóvenes han incursionado en el hábito de fumar, por lo que es un período vulnerable en lo que a este aspecto se refiere.

La investigación sobre la prevalencia de dependencia de la nicotina entre enfermeros, docentes y alumnos de enfermería de una universidad brasileña reveló que $30 \%$ había usado alguna vez el tabaco, $16 \%$ eran exfumadores, $14 \%$ eran fumadores, $70 \%$ nunca fumaron, $74 \%$ fumaban durante horas laborales y $47 \%$ intentaron dejar de fumar( ${ }^{(8)}$. Este estudio permite tener una base para futuras aproximaciones cualitativas sobre el comportamiento del fumador, lo cual ayudará a planear acciones preventivas en educación sobre este tema.

Otro estudio se propuso investigar, como los fumantes hacen el uso del simbolismo del cigarrillo y cognitivamente, qué significa fumar para ellos. Se 
desarrollaron entrevistas con el enfoque biográfico a fumadores de bajo nivel en dos universidades. Por medio de las narrativas de la historia de transición de la enseñanza secundaria a la universidad y a través de discutir el rol que el fumar jugó en sus biografías. Se obtuvieron tres categorías en relación a los contextos en los que se fuma: fiestas, estrés y aburrimiento. Los estudiantes usan el tabaco para lograr metas interrelaciónales y para estructurar su tiempo social y el espacio que podría de otra manera ser ambiguamente definido. Conceptualizando las actividades como juego, se obtienen valiosos hallazgos en etapas tempranas y las trayectorias del uso de tabaco entre estudiantes universitarios $^{(9)}$.

Son pocos los estudios que han explorado el significado de fumar en universitarios y el rol que esto puede jugar en la estructuración de la propia identidad. Muy poco es conocido sobre cómo el fumar es usado para facilitar las interacciones sociales dentro de la universidad y cómo los cigarrillos pueden servir como un recurso durante tiempos de dificultad emocional. Fumar entre los universitarios necesita situarse y abordarse dentro de un contexto social y cultural.

El objetivo de este estudio es conocer los significados atribuidos al consumo de tabaco entre estudiantes recién ingresados a la universidad.

\section{Método}

Se realizó un estudio de tipo cualitativo sobre la comprensión de cómo se han configurado los significados que los estudiantes recién ingresados atribuyeron al consumo de tabaco. Este tipo de estudio se caracteriza por la flexibilidad, es decir, puede ajustarse a conocimientos emergentes; él renuncia a la idea generalista, y camina hacia la construcción del universo particular del objeto de estudio( ${ }^{(9)}$.

Como metodología se propuso el interaccionismo simbólico, el cual se reconoce como una ciencia interpretativa cuyo propósito es el representar y comprender los procesos de creación y atribución de significados del mundo - de la realidad vivida. Se refiere a una compresión de actores particulares, en un espacio geográfico determinado, en situaciones específicas y en tiempos particulares(10).

Los participantes fueron 44 estudiantes recién ingresados a la universidad Pedagógica Nacional Francisco Morazán de Tegucigalpa, Honduras cursando la asignatura de Psicología Educativa y del Desarrollo, requisito de formación pedagógica para todo el alumnado.
El número de estudiantes fue definido por el criterio de saturación de la información. Como criterios de inclusión se definieron los siguientes: estudiantes matriculados por primera vez en el I y II período académico de 2008, inscritos en carreras de ambas facultades (Humanidades o Ciencia y Tecnología), del área urbana y rural.

Se procuró contar con igual número de hombres y mujeres a través de una selección aleatoria empleando para ello los listados de matrícula de la asignatura. Se identificaron aquellos estudiantes que eran fumadores y los que nunca habían fumado. Se empleó la técnica de grupos focales, cuyo propósito es generar discusión en un grupo de informantes guiados por un facilitador o moderador. En este estudio, se organizaron cuatro grupos focales (dos grupos de 10 y dos de 12 estudiantes) basados en los criterios de inclusión, así como en el género y uso del tabaco. La intención inicial era tener igual número de estudiantes en cada grupo pero razones de enfermedad y demanda académica limitó la asistencia. Los grupos focales se organizaron de la siguiente manera: a) Grupo 1: Mujeres fumadoras, b) Grupo 2: Mujeres no fumadoras, c) Grupo 3: Hombres fumadores, d) Grupo 4: Hombres no fumadores. Ninguno de los estudiantes se recusó a participar ni se retiro antes de tiempo de duración del grupo focal. El número de grupos focales fue definido por el criterio de saturación de la información. Se realizó una sola entrevista con cada grupo focal.

A continuación se procedió a la trascripción literal de la información obtenida en los grupos focales realizando el análisis respectivo, después de seguir el criterio de saturación de la información. Se entrenó a ayudantes de investigación para tomar anotaciones y apoyar el desarrollo de las entrevistas.

Los estudiantes fueron informados acerca del propósito del estudio; se tomaron cuidados en la confidencialidad y el manejo de la información brindada; los participantes firmaron un consentimiento informado. Se empleó la técnica de grupos focales cuyo propósito es generar discusión en un grupo de informantes guiados por un facilitador o moderador, para ello se elaboró un guión de entrevista semiestructurada que incluía preguntas acerca de los hábitos de consumo de tabaco de familiares y amigos, la comodidad versus la incomodidad de estar rodeado de personas que fuman, los motivos para fumar o no fumar en los universitarios, la influencia de la publicidad sobre elección de fumar y la historia personal del uso del tabaco. El proyecto fue aprobado por la Dirección de Investigación de la Universidad Pedagógica Nacional Francisco Morazán. 
El investigador responsable entrenó, a los ayudantes de investigación, para realizar las entrevistas entre ambos, para que tomaran anotaciones y para que apoyaran el desarrollo de las mismas. Se llevó a cabo una primera entrevista con el primer grupo focal, se grabó dicha reunión así como la transcripción de los datos y se realizó el análisis respectivo; posteriormente, se procedió a realizar la entrevista con el grupo $n^{\circ} 2$, transcribir y analizar y así se procedió con el resto de los grupos hasta agotar la información y cumplir el criterio de saturación de la información, identificándose temas relevantes desde la perspectiva de los estudiantes.

\section{Resultados}

Se detallan las categorías que sobresalieron y se presentaban como constantes en los distintos grupos focales, tomando como base el guión de preguntas de la entrevista.

\section{Hábitos de consumo de tabaco de familiares y amigos}

a) Familiares: se encontró una amplia variedad de respuestas desde "el que no se fuma en la familia" hasta familiares que "se la pasan fumando", padres que fuman "como una chimenea". Se encontró presencia significativa de tíos, abuelos, primos y hermanos que fuman. Algunos optan por fumar para mantener su peso y evitar aumentarlo. Existe una historia de consumo de tabaco de los padres por un periodo de más de 25 años, los que finalmente optaron dejar de fumar por aspectos de salud. Un estudiante reportó que uno de sus familiares usaba la frase "encendiendo el carro, encendiendo el cigarrillo" implicando lo automático del hábito de fumar. Se informaron problemas de tos, problemas de voz; algunos familiares fumaron toda su vida y algunos murieron de problemas en los pulmones.

b) Amigos: Informaron de amigos que fumaban un paquete de cigarrillos, eran compañeros a los cuales "les encantaba" fumar. Los amigos informaban que se sentían una "sequía" y fumaban exageradamente. Los estudiantes varones informaron un incremento en el número de amigas fumadoras y cómo en muchas ocasiones ellas les presionaban para fumar.

\section{Historia personal de consumir o no tabaco}

a) Consumo: Se fumaba por desahogo, para liberar estrés, o por encajar en el grupo. Existe una sensación de agrado por fumar, de sentir "sed" por el cigarrillo. Algunos participantes consideraron que fumaban exageradamente y otros declaraban que sentían que podían dejarlo pero que ya se habían acostumbrado a fumar.

b) No consumo: por motivos de salud (problemas en la garganta y vías respiratorias), voluntad de dejar de hacerlo, no les gustaba el olor del tabaco, del humo. Existía la preferencia a practicar un deporte, cuidar la salud física, no les llamaba la atención, implicaba en un gasto de dinero para "introducir" al organismo, por temor al castigo de los padres, existió una decisión para dejarlo, se promovía un ambiente libre de tabaco en su casa, para dar un buen ejemplo a los hijos, por principios de vida. Una decisión de no fumar motivada por sus estudios de formación para ser educador.

\section{Sensaciones al estar con personas que fuman}

a) Incomodidad: en los no fumadores este fue un aspecto importante, pues a los hijos les incomodaba que los padres fumasen estando ellos presentes. Los estudiantes fumadores informaron que al iniciar el consumo de tabaco les incomodaba estar con otros que fumaban aun cuando ellos lo hacían. Una estudiante expresó que ahora que es madre está considerando el dejar de fumar por su hijo recién nacido. Informaron sentirse incomodados en los lugares de trabajo especialmente en los espacios compartidos en donde las personas fumaban, siendo un olor que los ofende. En restaurantes o discotecas les incomoda por el hecho que la ropa queda impregnada de dicho olor y el humo provoca dolor de cabeza. Se percibe como dañino.

En una relación de noviazgo, afectaba el hecho que la novia fumase, sentía que su ropa y aliento estaban impregnados a tabaco y el novio sentía temor de ser quemado por el cigarrillo. Existe un malestar por la sensación del humo en la cara. En el caso de compartir el cuarto con su hermano fumador se sienten que el olor del tabaco ha saturado la habitación, reconocen el efecto del humo para los fumadores pasivos (los que reciben el humo del fumador) y eso molesta y resulta ser incómodo.

b) No incomodidad: los estudiantes no fumadores expresaron que el estar con compañeros o laborando con individuos que fuman, estando ellos presentes, no les incomodaba ni ofendía.

\section{Motivos que los estudiantes universitarios tiene para fumar}

La influencia de amigos, un ambiente diferente, el contagio y la vergüenza que sienten al rechazar una 
invitación para fumar. El fumar se reportó como un estimulante para estar despierto, y se fuma porque agrada. Uno de los motivos de peso en el caso de los varones, fue mostrar su masculinidad, por un prejuicio de no ser aceptado sino se fumaba. Muchos ya son fumadores desde el colegio y llegan a la universidad con ese hábito, se fumaba también como un "escape" para olvidarse de los problemas. Se hacía sólo para "decir que se fumaba" y así se comenzaba. El estrés en el proceso de estudios universitarios anima a fumar, a desear relajarse un rato, y encajar en el grupo. El "estar a la moda" se declaró como un motivo para fumar; el participar en reuniones familiares o entre amigos hace que, por imitación, se sientan incentivados a fumar y por el deseo de "relajarse".

\section{Motivos para no fumar en estudiantes universitarios}

El hecho de estar en una universidad que "forma profesores", y que posteriormente irá a formar a otros, se convierte en un incentivo para no fumar, dado que como profesor, será un ejemplo para sus estudiantes. Se declaró la formación obtenida en su familia de origen, continuar las reglas establecidas en su hogar, por salud, por fuerza de "voluntad de uno mismo", por metas propuestas ("no lo haré y lo cumple"), por principios morales, producto de un cambio espiritual (temor a Dios). Por las sensaciones de incomodidad, problemas de tos y poca tolerancia al olor del cigarro.

Además, el deseo de prevenir enfermedades y evitar las consecuencias de consumir tabaco. La universidad inculca ciertos valores encaminados a evitar los vicios, existe mayor control, mayor respeto al no fumar.

\section{Influencia de la publicidad en los hábitos de consumo en los estudiantes}

Los anuncios resultan atractivos, aparecen personas divirtiéndose, alegres, con atractivo sexual. Las escenas de los anuncios son muy agradables para el consumidor, se anima a ser imitador y a consumir tabaco, aparecen mujeres con escasa ropa que se vuelven un atractivo, pues se piensa que al fumar se podrá tener acceso a una joven como la del anuncio. La advertencia que aparece en el anuncio es en letra bastante pequeña que muchas veces no se percibe.

\section{Discusión}

El estudio pretendía obtener conocimiento de los significados atribuidos al consumo de tabaco de estudiantes recién ingresados a la universidad. Al ingresar a la universidad, los estudiantes de primer semestre se ven expuestos a las influencias de los estudiantes de semestres superiores, de los docentes y del personal administrativo, que pueden originar la adquisición de costumbres y hábitos que no tenían ${ }^{(11)}$.

A partir de la lectura se evidenciaron cinco categorías de creencias mediante las cuales los estudiantes recién ingresados construyen el significado acerca del consumo de tabaco.

\section{Presencia del consumo de tabaco en sus familias de origen y en sus amigos}

El uso de tabaco está presente en la mayoría de las familias y en el círculo cercano de amigos que se va formando en la universidad, lo que los ha animado a iniciarse en el consumo de tabaco; además, su uso les ha provocado pérdidas de miembros por razones de salud o procesos largos y dolorosos de enfermedad. El entorno familiar es un elemento importante en el consumo de tabaco, los integrantes de algunas familias por diferentes motivos no han podido responder adecuadamente al rol que les correspondía desempeñar en la misma. De igual modo la presión del grupo de pares puede tener influencia para efectos de su consumo(11-13).

\section{Decisiones personales para consumir tabaco}

La decisión por fumar guarda relación con lo informado en la literatura. Las razones más importantes para adquirir el hábito de fumar son: grado de placer, curiosidad, influencia de pares, rebeldía, insatisfacción con el medio que les rodea y familia. En cuanto a la toma de decisión por no fumar se incluye el que no les agrada el cigarrillo en sí, no les gusta porque daña la salud y/o no fuman porque les importa la opinión de la familia y de sus pares ${ }^{(14)}$.

En el caso de la universidad donde se desarrolló la investigación marcó mucho el que fuese una universidad pedagógica, formadora de formadores. Para los estudiantes el rol del profesor tiene un peso sustancial dado que lo consideran como una persona que es un modelo y que debe dar el ejemplo a sus alumnos. Por tanto, la visión de sí mismos como futuros educadores fue algo que se mostró como relevante en las entrevistas para su decisión de no consumir tabaco.

Es importante destacar que hay una diferencia importante entre los jóvenes que fumaban y los que no lo hacían, se trata del conocimiento del daño ocasionado por el tabaquismo. Los que no fumaban tenían mejor conocimiento al respecto(11-13). 


\section{Establecimiento de relaciones con personas que} consumen tabaco

La mayor parte de los estudiantes entrevistados evidenciaron incomodidad en la presencia de personas que fumaban. El tabaco no es peligroso sólo para quien lo consume en forma de cigarrillo fumado, lo es también para quienes inhalan las sustancias procedentes de del humo del tabaco presente en el ambiente(1).

\section{Incentivos para ser consumidor de tabaco}

Se destaca el contar con familiares o amigos fumadores. Esto está en acuerdo con estudios revisados que reportan que el tener padres, hermanos o amigos que fuman, así como el tener la opinión de que es definitiva o probablemente fácil dejar de fumar una vez que se ha empezado, constituyen factores asociados al consumo frecuente de tabaco entre los estudiantes universitarios ${ }^{(4)}$.

Las motivaciones para no ser consumidores de tabaco incluyen respuestas similares con las encontradas en otros estudios que reportan que el autocontrol (que no los atrae, que no les provoca el deseo de fumar, que no desean perder el control sobre sus vidas) y la presión social (el control ejercido por otros y el no quererse diferenciar de otros) son motivos muy importantes para su decisión(15). La literatura señala que las motivaciones son variables importantes a considerar cuando se desea prevenir el consumo de tabaco, protegiendo al individuo expuesto al riesgo y entregándole información sistemática y actualizada sobre los daños que el tabaco puede producir en su salud(11,14).

\section{La publicidad como factor de influencia en el consumo de tabaco}

Un ejemplo de insuficiencia en las medidas de control del tabaquismo es el referido a la publicidad. Muchos países desarrollados han logrado la total eliminación de la publicidad de varios medios de comunicación; por otro lado, en la mayoría de los países en desarrollo las medidas existentes se refieren a limitaciones al contenido de los anuncios, a los lugares donde estos pueden ser expuestos, al tiempo durante el cual pueden aparecer y a los horarios de presentación en radio y televisión. Sin embargo, se sabe que las limitaciones y las prohibiciones parciales tienen un efecto muy bajo en la reducción de consumo(13,16).

En el estudio, la televisión resultó el medio publicitario más nombrado, con alusión a elementos de explotación sexual particularmente de mujeres como figuras atrayentes para animarse a ser consumidor de tabaco y como elemento de afirmación de masculinidad.

\section{Conclusiones}

El estudio permitió un acercamiento a la vivencia del estudiante recién ingresado a la universidad en lo que se refiere a los significados que ha construido sobre el consumo de tabaco. Algunas reflexiones al respecto:

El espacio universitario puede convertirse en un factor de riesgo o un factor protector en el consumo de tabaco. La experiencia en la aplicación de estrategias educativas para la prevención del consumo de drogas ha demostrado que no es suficiente dar a conocer a la población los peligros del consumo. Es igualmente importante desarrollar en las personas la capacidad para resistir las presiones negativas de los pares y la publicidad, promoviendo su autocontrol, autoestima, auto-eficacia y autocuidado.

La Universidad Pedagógica Nacional Francisco Morazán como formadora de formadores posee un papel protagónico, potenciando la figura del profesor como modelo vivo a seguir. Esto resultó un elemento muy importante para los estudiantes, el mismo que podría tomarse un eje transversal en los procesos de enseñanza-aprendizaje y en los valores a los que se adscribe dicha institución educativa.

Es impostergable consolidar como línea de investigación el estudio de significados sobre el consumo de tabaco en estudiantes universitarios, de distintos años de avance académico, para comparar sus creencias y prácticas en relación al consumo de tabaco y establecer programas preventivos encaminados a fortalecer las decisiones de aquellos estudiantes que no fuman $y$ animar a los que fuman a dejar de hacerlo.

\section{Agradecimientos}

Agradecemos a la Comisión Interamericana para el Control del Abuso de Drogas/CICAD de la Secretaria de Seguridad Multidimensional/SSM de la Organización de los Estados Americanos/OEA, la Secretaria Nacional de Políticas sobre Drogas/SENAD do Gabinete de Seguridad Institucional/Brasil, la Escuela de Enfermería de Ribeirao Preto de la Universidad de Sao Paulo y Centro Colaborador de la Organización Mundial de la Salud para el Desarrollo de la Investigación en Enfermería, la población representada en los estudios de investigación, bien como a las autoridades de las universidades representadas por los participantes del Programa En-Line de Especialización en Investigación sobre el Fenómeno de las Drogas, periodos 2006, 2007, 2008 y 2009. 


\section{Referencias}

1. Organización Mundial de la Salud. Convenio Marco de la OMS para el control del tabaco. Lima: SINCO Editores; 2005. p. 38.

2. Zárate $M$, Zavaleta A, Danjoy D, Chanamé E, Prochazka $R$, Salas $M$, et al. Practicas de consumo de tabaco $y$ otras drogas en estudiantes de ciencias de la salud de una universidad privada de Lima, Perú. Universidad de Antioquia. Facultad de Enfermería. Invest Educ Enferm. 2006;25(2):72-81.

3. Goodman LS, Gilman A. Las bases farmacológicas de la terapéutica. 8. ed. Argentina: Panamericana; 1991.

4. Zarate M, Zavaleta A, Dajov D, Chanamé E, Prochazka $R$, Salas $M$, Maldonado V. Practicas de consumo de tabaco y otras drogas en estudiantes de ciencias de la salud de una universidad privada de Lima, Perú. Invest Educ Enferm. 2006;24(2):72-81.

5. Diario El Heraldo. Informe noticioso. 19 de junio de 2004. [acesso 20 dezembro 2007]. Disponível en: http://www.elheraldo.hn/nota. php?nid=14321\&sec=12\&fecha=2004-06-19.

6. Consuegra RVG, Zago MMF. Creencias en fumadores pertenecientes a un programa de salud cardiovascular. Rev. Latino-Am. Enfermagem 2004 marzo-abril, 12(n. esp):412-9.

7. Stromberg $P$, Nichter $M$, Nichter $M$. Taking play seriously: Low-level smoking among college students. Cult Med Psychiatry 2007;31(1):1-24.

8. Pillon SC, Ramos R, Dunn J. O comportamento do fumar entre enfermeras. Acta Paul Enferm. 2002,15(2):6570.

9. Alvarez JL, Jurgenson G. Como hacer investigación cualitativa. Fundamentos y Métodos. México DF: Paidós; 2003.

10. Blumer $\mathrm{H}$. Simbolic interactionism: perspective end method. Berkeley: University of California; 1969.

11. Tafur LA, Ordonez GA, Millan JC, Varela JM, Rebellon P. Tabaquismo en personal de la Universidad Santiago de Cali. Colombia Med. 2005;36(3):194-8.

12. Henriquez PC Carvalho, AMP. Percepção dos benefícios do consumo de drogas e das barreiras para seu abandono entre estudantes da área da saúde. Rev. Latino-Am. Enfermagem. 2008 jul-ago, 16(número especial):621-6.

13. Ortega NM, Osório EA, Pedrão LJ. El significado de drogas para el estudiante de enfermería según el modelo de creencias en salud de Rosenstock. Rev. Latino-Am. Enfermagem. 2004 marzo-abril, 12(número especial):316-23.
14. González L, Berger K. Consumo de tabaco en adolescentes: Factores de riesgo y factores protectores. Cienc Enferm. 2002;8(2):27-35.

15. Behn V, Cruz M, Huaiquian J, Naveas R, Sotomayor $\mathrm{H}$. Motivaciones de no fumadores para continuar esta conducta saludable. Cienc Enferm. 2003;9(1):131-7. 16. Chaloupka J P. Tobacco control in developing countries. New York: Oxford University Press; 2000. 